\title{
Short-term beat-to-beat variability of the QT interval is increased and correlates with parameters of left ventricular hypertrophy in patients with hypertrophic cardiomyopathy
}

\begin{tabular}{|c|c|}
\hline Journal: & Canadian Journal of Physiology and Pharmacology \\
\hline Manuscript ID: & cjpp-2014-0526.R1 \\
\hline Manuscript Type: & Article \\
\hline Date Submitted by the Author: & 21-Apr-2015 \\
\hline Complete List of Authors: & $\begin{array}{l}\text { Orosz, Andrea; University of Szeged, Department of Pharmacology and } \\
\text { Pharmacotherapy } \\
\text { Baczkó, István; University of Szeged, Department of Pharmacology and } \\
\text { Pharmacotherapy } \\
\text { Nagy, Viktória; University of Szeged, 2nd Department of Internal Medicine } \\
\text { and Cardiology Centre } \\
\text { Gavallér, Henriette; University of Szeged, 2nd Department of Internal } \\
\text { Medicine and Cardiology Centre; Diagnoscan Hungary Ltd., } \\
\text { Csanády, Miklós; University of Szeged, 2nd Department of Internal } \\
\text { Medicine and Cardiology Centre } \\
\text { Forster, Tamás; University of Szeged, 2nd Department of Internal Medicine } \\
\text { and Cardiology Centre } \\
\text { Papp, Julius; University of Szeged, Department of Pharmacology and } \\
\text { Pharmacotherapy; Hungarian Academy of Sciences, MTA-SZTE Research } \\
\text { Group of Cardiovascular Pharmacology } \\
\text { Varró, András; University of Szeged, Department of Pharmacology and } \\
\text { Pharmacotherapy; Hungarian Academy of Sciences, MTA-SZTE Research } \\
\text { Group of Cardiovascular Pharmacology } \\
\text { Lengyel, Csaba; University of Szeged, 1st Department of Internal Medicine } \\
\text { Sepp, Róbert; University of Szeged, 2nd Department of Internal Medicine } \\
\text { and Cardiology Centre }\end{array}$ \\
\hline Keyword: & $\begin{array}{l}\text { hypertrophic cardiomyopathy, QT prolongation, QT dispersion, Tpeak-Tend } \\
\text { interval, short-term beat-to-beat variability of the QT interval }\end{array}$ \\
\hline
\end{tabular}

\section{SCHOLARONE \\ Manuscripts}




\section{Short-term beat-to-beat variability of the QT interval is increased and correlates with parameters of left ventricular hypertrophy in patients with hypertrophic cardiomyopathy}

Orosz, Andrea; Baczkó, István; Nagy, Viktória; Gavallér, Henriette; Csanády, Miklós; Forster, Tamás; Papp, Julius Gy.; Varró, András; Lengyel, Csaba; Sepp, Róbert

A. Orosz, I. Baczkó, J. Gy. Papp, A. Varró. Department of Pharmacology and Pharmacotherapy, University of Szeged, Hungary.

V. Nagy, H. Gavallér, M. Csanády, T. Forster, R. Sepp. $2^{\text {nd }}$ Department of Internal Medicine and Cardiology Centre, University of Szeged, Hungary

H. Gavallér. Diagnoscan Hungary Ltd.

J. Gy. Papp, A. Varró. MTA-SZTE Research Group of Cardiovascular Pharmacology, Hungarian Academy of Sciences, Szeged, Hungary

Cs. Lengyel. $1^{\text {st }}$ Department of Internal Medicine, University of Szeged, Hungary

\section{Corresponding author:}

Róbert Sepp, M.D., Ph.D.

$2^{\text {nd }}$ Department of Internal Medicine and Cardiology Centre,

Faculty of Medicine, University of Szeged

Korányi fasor 6., 6720 Szeged, Hungary

Phone: $+36.62 .545-220$

Fax: $+36.62 .544-568$

e-mail: sepp.robert@med.u-szeged.hu 


\section{$\underline{\text { Abstract }}$}

Stratification models for prediction of sudden cardiac death (SCD) are inappropriate in patients with hypertrophic cardiomyopathy (HCM). We investigated conventional ECG repolarization parameters and the beat-to-beat short-term QT interval variability (QT-STV), a new parameter of proarrhythmic risk, in 37 patients with HCM (21 males, average age $48 \pm 15$ years). Resting ECGs were recorded for 5 minutes and the frequency corrected QT interval (QTc), QT dispersion (QTd), beat-to-beat short-term variability of QT interval (QT-STV) and the duration of terminal part of $\mathrm{T}$ waves (Tpeak-Tend) were calculated. While all repolarization parameters were increased significantly in patients with HCM compared to controls (QTc: $488 \pm 61$ vs. $434 \pm 23 \mathrm{~ms}, \mathrm{p}<0.0001$; QT-STV: $4.5 \pm 2$ vs. $3.2 \pm 1 \mathrm{~ms}, \mathrm{p}=0.0002$; Tpeak-Tend duration: $107 \pm 27$ vs. $91 \pm 10 \mathrm{~ms}, \mathrm{p}=0.0015$; QTd: $47 \pm 17$ vs. $34 \pm 9 \mathrm{~ms}, \mathrm{p}=0.0002$ ), QT-STV had the highest relative increase (+41\%). QT-STV also showed the best correlation with indices of left ventricular hypertrophy, i.e. maximal left ventricular (LV) wall thickness normalized for body surface area $(\mathrm{BSA} ; \mathrm{r}=0.461, \mathrm{p}=0.004)$ or LV mass (determined by cardiac magnetic resonance imaging) normalized for BSA $(\mathrm{r}=0.455, \mathrm{p}=0.015)$. In summary, beat-to-beat QT-STV showed the most marked increase in patients with HCM and may represent a novel marker which merits further testing for increased SCD risk in HCM.

Key words: hypertrophic cardiomyopathy, repolarization, QT prolongation, QT dispersion, Tpeak-Tend interval, short-term beat-to-beat variability of the QT interval, left ventricular hypertrophy, sudden cardiac death 


\section{Introduction}

Hypertrophic cardiomyopathy $(\mathrm{HCM})$ is a common inherited cardiac disease with a prevalence of one in 500 in the general population (Maron et al. 1995), characterized by marked but variable left ventricular hypertrophy and myocardial fibrosis (Gersh et al. 2011). HCM is associated with lethal ventricular arrhythmias (Gersh et al. 2011; Maron 2002; Wigle et al. 1995), and it is the most common cause of sudden cardiac death (SCD) in young individuals (Decker et al. 2009; Maron 2010) and in competitive athletes younger than 35 years (Maron et al. 2009). The reliable assessment of SCD risk in individual HCM patients and the identification of patients for implantable cardioverter defibrillator (ICD) implantation, the most effective intervention for SCD prevention, are critically important. Currently models for SCD risk stratification uses non-invasive conventional clinical markers including family history of SCD, unexplained recent syncope, multiple repetitive non-sustained ventricular tachycardia identified on ambulatory ECG, abnormal blood pressure response upon exercise and massive ( $\geq 30 \mathrm{~mm}$ thickness) LV hypertrophy (Gersh et al. 2011; Maron 2010; Monserrat et al. 2003; Spirito et al. 2009; Spirito et al. 2000). Additional risk modifier factors that help making the decision regarding ICD implantation include marked left ventricular outflow tract obstruction, high risk genotype involving multiple sarcomere mutations, extensive and diffuse late gadolinium enhancement on MRI, apical aneurism in the left ventricle, and coronary artery disease (Maron 2010). However, all established SCD risk factors exhibit a low positive predictive value and the current SCD risk assessment algorithm in HCM is still considered incomplete and hampered by lack of sufficient evidence for all elements (McKeown and Muir 2013). This notion is supported by SCD events in HCM patients who were not considered to be at high risk for SCD (Maron et al. 2008).

ECG parameters have been studied for their utility as prognostic non-invasive markers in SCD risk assessment in HCM. The prolongation of the frequency corrected QT interval 
(QTc) and increased QTc dispersion (characterizing spatial repolarization heterogeneity) were observed in patients with HCM (Buja et al. 1993; Dritsas et al. 1992; Yi et al. 1998). However, QTc prolongation and QTc dispersion have been found not to be predictive for SCD in HCM patients (Maron et al. 2001; Yi et al. 1998). The Tpeak-Tend interval, another ECG parameter representing spatial (including transmural) dispersion of repolarization (Antzelevitch 2001), has been shown to more reliably predict the development of Torsades des Pointes (TdP) polymorphic ventricular tachycardia in congenital (Schwartz et al. 2001) and acquired long QT syndromes than QTc prolongation or increased QT dispersion (Yamaguchi et al. 2003). The prolongation of the Tpeak-Tend interval has been associated with SCD in the Oregon Sudden Unexpected Death Study (Panikkath et al. 2011). In a small number of HCM patients with a cardiac troponin I gene mutation, the Tpeak-Tend interval was associated with SCD (Shimizu et al. 2002). In addition to increased spatial dispersion of repolarization, increased temporal variability of cardiac ventricular repolarization has been associated with increased propensity for ventricular arrhythmias and SCD in patients with different cardiovascular pathologies (Atiga et al. 1998; Haigney et al. 2004; Piccirillo et al. 2007). The beat-to-beat short-term temporal variability of the QT interval (QT-STV) has been suggested as a novel ECG parameter for more reliable prediction of development of serious ventricular arrhythmias both in experimental animal and clinical studies [for a current review see (Varkevisser et al. 2012)]. However, according to our knowledge, the characterization of QT-STV in patients with HCM has not been addressed in the literature so far.

Therefore, the aim of the present study was to compare conventional ECG parameters of repolarization and QT-STV in HCM patients and age matched healthy volunteers, and to investigate whether there was a relationship between QT-STV and the morphologic parameters of cardiac hypertrophy, proved to be predictors of higher SCD risk, obtained by cardiac echocardiography and MRI in patients with HCM. 


\section{Methods}

\section{Ethics Statement}

The studies described here were carried out in accordance with the Declaration of Helsinki (2000) of the World Medical Association and were approved by the Scientific and Research Ethical Committee of the Medical Scientific Board at the Hungarian Ministry of Health (ETTTUKEB), under ethical approval No. 4987-0/2010-1018EKU (338/PI/010).

\section{Study Subjects}

Thirty-seven consecutive patients with hypertrophic cardiomyopathy (HCM) were enrolled into the study. The diagnosis of HCM was based on established diagnostic criteria (Gersh et al. 2011). Among the $37 \mathrm{HCM}$ patients, 24 patients were taking beta blockers and 8 patients were taking verapamil as first line therapy. Three patients were taking cardiac medications known to prolong QT interval (two were taking amiodarone and one was taking propafenone). None of the patients were on any other drug therapy with known QT-interval prolonging effect.

A total of 37 age- and gender-matched healthy volunteers (mean age $43 \pm 12$ years, males/females 21/16) without a history or evidence of heart disease was enrolled in the study as controls. Body mass index was significantly lower in the control group ( $25 \pm 4$ vs. $28 \pm 6$ $\mathrm{kg} / \mathrm{m}^{2}, \mathrm{p}=0.007$, see Table 1). All of the control individuals and HCM patients were of Caucasian origin.

\section{Electrocardiography}

12-lead electrocardiograms were continuously recorded for $5 \mathrm{~min}$ at rest, in the supine position to obtain signals with the least amount of motion artefact. In all leads the ECG signals were digitized at $2000 \mathrm{~Hz}$ sampling rate with a multichannel data acquisition system 
(Cardiosys A01 software, Experimetria Ltd., Budapest, Hungary; MDE Heidelberg GMBH, Heidelberg, Germany) connected to a PC and stored for later off-line analysis.

Out of the repolarization parameters we analyzed the frequency corrected QT interval $(\mathrm{QTc})$ using Bazett's $(\mathrm{QTc}=\mathrm{QT} / \sqrt{\mathrm{RR}})$, Fridericia $(\mathrm{QTc}=\mathrm{QT} /[\mathrm{RR} / 1000] 1 / 3)$, Framingham $(\mathrm{QTc}=\mathrm{QT}+[0.154 *\{1000-\mathrm{RR}\}])$ and the Hodges formulas $(\mathrm{QTc}=\mathrm{QT}+1.75 *[60$ 000/RR-60]), the QT dispersion (QTd), the PQ and QRS intervals, the duration of terminal part of T waves (Tpeak-Tend) and the short-term variability of QT interval (QT-STV).

The RR, QT intervals and duration of the T wave from the peak to the end (TpeakTend) intervals were measured automatically in 30 consecutive beats (minimum number of intervals needed for variability measurements), were checked by the investigator and manually corrected if needed and were calculated as the average of 30 beats. Heart rate correction of QT intervals was performed by the Bazett's, Fridericia, Framingham and Hodges formulas, and QTc interval duration was defined as the mean duration of all QTc intervals measured. As no statistical difference was noted between different correction methods with regard to corrected QT interval, QTc corrected with the Bazett's formula was used for further comparisons. The PQ and QRS intervals were measured as the average of 15 consecutive beats. All measurements were carried out using lead II and in case of excessive noise in lead II, lead V5.

To characterize the temporal instability of beat-to-beat repolarization, Poincare' plots of the QT intervals were constructed, where each QT value is plotted against its former value. QT-STV was calculated using the following formula: QT-STV $=\sum\left|D_{n+1}-D_{n}\right| /(30 x \sqrt{ } 2)$, where D represents the duration of the QT intervals. This calculation defines the QT-STV as the mean distance of points perpendicular to the line of identity in the Poincaré plot.

Patients were excluded if they had history and/or clinical documentation of significant comorbidity [e.g. known coronary artery disease, severe obstructive lung disease, pulmonary 
embolism, primary pulmonary hypertension, valvular heart disease, pericardial disease, moderate-severe renal failure (serum creatinine $>2 \mathrm{mg} / \mathrm{dl}$ ), moderate-severe anemia (hemoglobin $<11 \mathrm{~g} / \mathrm{dl}$ )]. All patients with complete left bundle branch block, with rhythm other than normal sinus rhythm (e.g. atrial fibrillation, pacemaker-dependent rhythm), with excessive $(>5 \%)$ ectopic atrial or ventricular beats or with excessive noise on the electrocardiographic signal that precluded analysis of the ECG waveform, were also excluded. Patients were instructed not to consume significant amount of food within 3 hours, to drink alcohol, coffee or to smoke within 10 hours.

\section{Echocardiography}

All HCM patients and controls underwent transthoracic echocardiographic examination. Twodimensional echocardiographic images were obtained by a commercially available Toshiba Powervision 8000 echocardiography equipment, in a number of cross-sectional planes using standard imaging positions to determine standard morphological and functional parameters [left ventricular end-systolic diameter (LVESD), left ventricular end-diastolic diameter (LVEDD), ejection fraction (EF), left atrial diameter (LA), resting left ventricular outflow tract (LVOT) peak gradient]. Maximal left ventricular wall thickness (LVmax) was defined as the largest wall thickness of the left ventricle at any left ventricular segment. LVmax was also normalized for body surface area (LVmax BSA). Echocardiographic parameters for the HCM group are shown in Table 1.

\section{Cardiac MRI}

In all HCM patients, cardiac magnetic resonance imaging (MRI) was carried out to determine the left ventricular mass (LVM). MRI assessments were performed in supine position with the head first on a commercially available 1.5T scanner (Signa Excite HDxT, GE Medical 
Systems) using a phased-array body coil. Sequential gradient-echo short-axis cine images (base to apex; slice thickness: $8 \mathrm{~mm}$; field of view: $43 \mathrm{~mm}$; matrix: 224x224; repetition time: 100 milliseconds) covering the entire LV were acquired during breath hold after normal expiration. Three long-axis images (2-, 3- and 4-chamber views) were also acquired. The acquisition was triggered by ECG. The gradient-echo short-axis images were used to measure LVM by planimetry of the manually defined endocardial and epicardial borders on each short-axis image covering the entire LV. The measurement was performed in both end diastole and end systole to enable calculation of LV ejection fraction. Papillary muscles were not included in the LVM. LVM was also normalized for body surface area (LVM BSA).

\section{Statistics}

All data are expressed as mean \pm standard deviation (SD). Comparisons between HCM patients and controls for the study variables were done using the independent samples Student's $t$ test for normally distributed parameters. Normal distribution was verified by the KolmogorovSmirnov test. Degree of association between two variables was expressed by the Pearson correlation coefficient (r). The statistical analyses were performed using the MedCalc software package (ver. 14.12.0). Statistical significance was accepted at the $\mathrm{p}<0.05$ level. 


\section{Results}

\section{Electrocardiographic parameters in HCM patients and controls}

Comparison of ECG parameters between HCM patients and controls are presented in Table 2. Patients with HCM exhibited significantly increased RR, PQ and QRS intervals. QTc was significantly prolonged in HCM patients (Figure 1, A), regardless of the method used for QTc correction (using Bazett's, Fridericia, Framingham or the Hodges formulas). There was no difference between QTc intervals in the HCM group obtained by the different correction methods. The terminal part of the $\mathrm{T}$ wave, the Tpeak-Tend interval was also markedly longer in HCM patients (Figure 1, B). QT dispersion (Figure 1, C) and short-term QT variability was also markedly increased in patients with HCM (Figure 1, D). The largest relative increase among the different parameters was seen with regard to short-term QT variability with a relative increase of $41 \%$. Differences between the HCM and control groups remained highly significant when we excluded patients taking QT prolonging drugs (amiodarone or propafenone, $n=3$ ) from the comparisons. BMI or obesity status did not correlate with either of the repolarization parameters.

\section{Correlation of repolarization parameters in HCM patients}

Correlation between different repolarization parameters, QT dispersion and short-term QT variability are given in Table 3. The QTc prolongation correlated significantly with the prolongation of the Tpeak-Tend interval, but not the QRS width, indicating that the QTc prolongation was, at least in part, due to the prolongation of the terminal phase of the $\mathrm{T}$ wave. Short-term QT variability showed a relatively strong correlation with the QTc prolongation and with, to a lesser extent, with the Tpeak-Tend interval. The QT dispersion did not correlate with any of the repolarization parameters. 


\section{Correlation between repolarization parameters and echocardiographic parameters in}

\section{HCM patients}

Correlation between ECG repolarization parameters and echocardiography parameters in HCM patients showed no correlation between these parameters except for a weak correlation between short-term QT variability and left ventricular end-systolic diameter or left ventricular ejection fraction (data not shown).

\section{Correlation between repolarization parameters and indices of left ventricular hypertrophy in HCM patients}

Correlation between repolarization parameters and indices of left ventricular hypertrophy (maximal left ventricular wall thickness and left ventricular mass, measured by cardiac magnetic resonance imaging) with or without normalization for body surface area are shown in Table 4. Degree of correlation increased with normalization in almost all comparisons. Short-term QT variability showed significant, albeit modest correlation, with both unnormalized and normalized indices of left ventricular hypertrophy (LVmax; LVmax BSA and LVM BSA, Figures 2 and 3). Tpeak-Tend interval also correlated significantly with some of the hypertrophy parameters, but showed no significant correlation to the most reliable hypertrophy parameter, i. e. LVM indexed for BSA. 


\section{Discussion}

In this study we showed that all ECG repolarization parameters, including frequency corrected QT interval (QTc), QT dispersion (QTd), beat-to-beat short-term variability of QT interval (QT-STV) and the duration of terminal part of $\mathrm{T}$ waves (Tpeak-Tend) were significantly increased in patients with HCM. QT-STV showed the largest relative increase among the different parameters and also showed the best correlation with indices of left ventricular hypertrophy, i.e. maximal left ventricular wall thickness or MRI derived LV mass, indexed or unindexed for body surface area.

Hypertrophic cardiomyopathy is characterized by morphological and structural changes, including left ventricular hypertrophy, myocardial fibrosis, myofiber disarray, and small vessel disease among them, that may represent an arrhythmogenic substrate of the disease (Maron 2002). Remodeling in HCM is a progressive process (Olivotto et al. 2012) and a very recent study highlighted a close correlation between the development of adverse remodeling and increased risk for SCD in HCM patients (Vriesendorp et al. 2014). In chronic heart failure, structural remodeling is accompanied by electrical remodeling that includes profound changes in the expression of voltage gated depolarizing and repolarizing ionic currents and exchangers resulting in decreased cardiomyocyte repolarizing capacity [for a comprehensive review see (Nattel et al. 2007)]. This decreased repolarizing capacity can be brought about by an increase in depolarizing currents $\left(\mathrm{Na}^{+}\right.$and $\left.\mathrm{Ca}^{2+}\right)$ and decreased potassium channel densities (particularly $\mathrm{I}_{\mathrm{K} 1}, \mathrm{I}_{\mathrm{to}}$ and $\mathrm{I}_{\mathrm{Ks}}$ ), resulting in action potential prolongation manifested as QT

prolongation on the surface ECG (Beuckelmann et al. 1993; Li et al. 2004; Tomaselli and Marban 1999). Increased action potential prolongation favors increased $\mathrm{Ca}^{2+}$ influx that in turn can facilitate delayed afterdepolarization (DAD) and arrhythmia development (Bers et al. 2006). Prolongation of repolarization can also precipitate serious ventricular re-entry type 
arrhythmias via promoting early afterdepolarization (EAD) generation (Michael et al. 2009; Zeng and Rudy 1995). A particularly interesting observation is the increase in slowly inactivating, late sodium current $\left(\mathrm{I}_{\mathrm{Na}, \text { late }}\right)$, that has been shown to prolong repolarization in heart failure and also to contribute to arrhythmogenesis (Valdivia et al. 2005). Most interestingly, these elements of arrhythmogenic electrical remodeling have not only been described in congestive heart failure and pathologies leading to cardiac hypertrophy, but a very recent study identified similar changes in cardiomyocytes isolated from HCM patients (Coppini et al. 2013). The decreased repolarization capacity due to HCM leads to markedly impaired repolarization reserve (Varro and Baczko 2011) and increased arrhythmia susceptibility in HCM, where even drugs or dietary constituents with only mild repolarization inhibitory effects can provoke serious ventricular arrhythmias and SCD.

Impaired repolarization reserve and temporal repolarization instability can be characterized by the calculation of the short-term beat-to-beat variability of the QT interval (QT-STV) which characterizes differences in the duration of QT intervals in consecutive heart beats. This parameter has emerged as a novel parameter for assessing pro-arrhythmia risk in arrhythmogenic cardiac diseases and has been shown to be a more reliable estimate and predictor of pro-arrhythmic risk associated with impaired repolarization reserve as opposed to more conventional ECG parameters of repolarization (Berger 2003; Varkevisser et al. 2012). A number of animal experimental and clinical studies (Hinterseer et al. 2009; Hinterseer et al. 2010; Lengyel et al. 2007; Thomsen et al. 2004; van Opstal et al. 2001) found that QT-STV was increased and showed a better correlation with subsequent arrhythmias than repolarization prolongation in animals or patients with decreased repolarization reserve later exhibiting serious ventricular arrhythmias and/or SCD. 
QT variability has been previously shown to be increased in patients with HCM. The normalized QT variability index (QTVI), measured as described by Berger et al, was shown to be higher in HCM patients than in controls (Atiga et al. 2000), and the greatest abnormality was detected in patients with malignant HCM mutations (i.e. Arg403Gln mutation of the beta myosin heavy chain gene). In a recent paper (Magri et al. 2014) several myocardial repolarization parameters, including normalized QT variability (QTVN) and QT variability index (QTVI) were shown to be associated with the presence and extent of late gadolinium enhancement (LGE) detected on cardiac magnetic resonance in patients with hypertrophic cardiomyopathy. Both QTVN and QTVI were higher in patients with LGE. Among other parameters, the extent of LGE and sudden cardiac death risk factor burden (the number of traditional risk factors for sudden cardiac death) predicted QTVI. Of note, left ventricular mass index was also associated with QTVN. However, QTVI or QTVN provide a measure of overall QT variability measured during the whole duration of the ECG recording and does not take into account beat-to-beat variations, which might be equally, or even more important.

In our work, QT-STV showed correlation with different indices of LV hypertrophy. Myocardial hypertrophy is an inherent feature of HCM, the magnitude of which is shown to be related to adverse cardiac events, including sudden cardiac death, in patients with HCM (Spirito et al. 2000). Indeed, pronounced myocardial hypertrophy, defined as left ventricular wall thickness $>30 \mathrm{~mm}$ is an independent predictor for SCD in HCM, and a prophylactic ICD implantation for primary SCD prevention is suggested in such a cases by current clinical guidelines (Gersh et al. 2011). Left ventricular mass, measured by MRI, might be an even stronger predictor for such adverse events, as markedly increased LV mass index was proved to be more sensitive with regard to HCM-related death, than maximal wall thickness (Olivotto et al. 2008). It is of note that ECG voltage parameters, indicating the magnitude of myocardial hypertrophy, also correlates with adverse events in HCM (Ostman-Smith et al. 2010). 
In summary, we have provided evidence that among ECG repolarization parameters, the beatto-beat short-term variability of QT interval showed a pronounced increase in patients with HCM. QT-STV also correlated with indices of left ventricular hypertrophy, known to be associated with SCD in HCM. We conclude that QT-STV may represent a novel candidate non-invasive marker which should be tested for increased SCD risk in patients with HCM.

\section{Study limitations:}

The study was not designed to assess link between QT variability and increased risk of sudden cardiac death risk. With this regard, it would be necessary to prove that increased QTSTV is directly linked to SCD risk in HCM. Initially, it would be important to show whether QT-STV correlates with other established parameters defining increased SCD risk (occurrence of syncope, abnormal blood pressure response during exercise, NSVT on 24-hour Holter recording, etc.). Further, a direct association of QT-STV with SCD should be tested in a large patient cohort with HCM in a multivariate analysis.

\section{Acknowledgement}

This work was supported by grants from the Hungarian Research Fund OTKA (NK-104331, K-109610), the National Development Agency and co-financed by the European Social Fund [TÁMOP-4.2.2A-11/1/KONV-2012-0073, TÁMOP-4.1.2.E-13/1/KONV-2013-0011, GOP1.1.1-11-2011-0081 and TÁMOP-4.2.2.A-11/1/KONV-2012-0035 (project title: "Interaction of environmental and genetic factors in the development of immunmediated and oncological diseases")], and the Hungarian Academy of Sciences.

The funders had no role in study design, data collection and analysis, decision to publish, or preparation of the manuscript. 


\section{Conflict of interest}

None. 


\section{References}

Antzelevitch, C. 2001. T peak-Tend interval as an index of transmural dispersion of repolarization. European Journal of Clinical Investigation 31(7): 555-557. PMID:11454006.

Atiga, W.L., Calkins, H., Lawrence, J.H., Tomaselli, G.F., Smith, J.M., and Berger, R.D. 1998. Beat-to-beat repolarization lability identifies patients at risk for sudden cardiac death. Journal of Cardiovascular Electrophysiology 9(9): 899-908. PMID:9786070.

Atiga, W.L., Fananapazir, L., McAreavey, D., Calkins, H., and Berger, R.D. 2000. Temporal repolarization lability in hypertrophic cardiomyopathy caused by beta-myosin heavy-chain gene mutations. Circulation 101(11): 1237-1242. PMID:10725281.

Berger, R.D. 2003. QT variability. Journal of Electrocardiology 36 Suppl: 83-87. PMID:14716597.

Bers, D.M., Despa, S., and Bossuyt, J. 2006. Regulation of $\mathrm{Ca} 2+$ and $\mathrm{Na}+$ in normal and failing cardiac myocytes. Annals of the New York Academy of Sciences 1080: 165-177. doi: 10.1196/annals. 1380.015. PMID:17132783.

Beuckelmann, D.J., Nabauer, M., and Erdmann, E. 1993. Alterations of K+ currents in isolated human ventricular myocytes from patients with terminal heart failure. Circulation Research 73(2): 379-385. PMID:8330380.

Buja, G., Miorelli, M., Turrini, P., Melacini, P., and Nava, A. 1993. Comparison of QT dispersion in hypertrophic cardiomyopathy between patients with and without ventricular arrhythmias and sudden death. The American Journal of Cardiology 72(12): 973-976. PMID:8213559.

Coppini, R., Ferrantini, C., Yao, L., Fan, P., Del Lungo, M., Stillitano, F., et al. 2013. Late sodium current inhibition reverses electromechanical dysfunction in human hypertrophic cardiomyopathy. Circulation 127(5): 575-584. doi: 10.1161/CIRCULATIONAHA.112.134932. PMID:24002721.

Decker, J.A., Rossano, J.W., Smith, E.O., Cannon, B., Clunie, S.K., Gates, C., et al. 2009. Risk factors and mode of death in isolated hypertrophic cardiomyopathy in children. Journal of the American College of Cardiology 54(3): 250-254. doi: 10.1016/j.jacc.2009.03.051. PMID:19589438.

Dritsas, A., Sbarouni, E., Gilligan, D., Nihoyannopoulos, P., and Oakley, C.M. 1992. QTinterval abnormalities in hypertrophic cardiomyopathy. Clinical Cardiology 15(10): 739-742. PMID:1395184.

Gersh, B.J., Maron, B.J., Bonow, R.O., Dearani, J.A., Fifer, M.A., Link, M.S., et al. 2011. $2011 \mathrm{ACCF} / \mathrm{AHA}$ guideline for the diagnosis and treatment of hypertrophic cardiomyopathy: a report of the American College of Cardiology Foundation/American Heart Association Task Force on Practice Guidelines. Circulation 124(24): e783-831. doi: 10.1161/CIR.0b013e318223e2bd. PMID:22068434. 
Haigney, M.C., Zareba, W., Gentlesk, P.J., Goldstein, R.E., Illovsky, M., McNitt, S., et al. 2004. QT interval variability and spontaneous ventricular tachycardia or fibrillation in the Multicenter Automatic Defibrillator Implantation Trial (MADIT) II patients. Journal of the American College of Cardiology 44(7): 1481-1487. doi: 10.1016/j.jacc.2004.06.063. PMID:15464332.

Hinterseer, M., Beckmann, B.M., Thomsen, M.B., Pfeufer, A., Dalla Pozza, R., Loeff, M., et al. 2009. Relation of increased short-term variability of QT interval to congenital long-QT syndrome. The American Journal of Cardiology 103(9): 1244-1248. doi: 10.1016/j.amjcard.2009.01.011. PMID:19406266.

Hinterseer, M., Beckmann, B.M., Thomsen, M.B., Pfeufer, A., Ulbrich, M., Sinner, M.F., et al. 2010. Usefulness of short-term variability of QT intervals as a predictor for electrical remodeling and proarrhythmia in patients with nonischemic heart failure. The American Journal of Cardiology 106(2): 216-220. doi: 10.1016/j.amjcard.2010.02.033. PMID:20599006.

Lengyel, C., Varro, A., Tabori, K., Papp, J.G., and Baczko, I. 2007. Combined pharmacological block of $\mathrm{I}(\mathrm{Kr})$ and $\mathrm{I}(\mathrm{Ks})$ increases short-term QT interval variability and provokes torsades de pointes. British Journal of Pharmacology 151(7): 941-951. doi: 10.1038/sj.bjp.0707297. PMID:17533421.

Li, G.R., Lau, C.P., Leung, T.K., and Nattel, S. 2004. Ionic current abnormalities associated with prolonged action potentials in cardiomyocytes from diseased human right ventricles. Heart Rhythm: the official journal of the Heart Rhythm Society 1(4): 460-468. doi: 10.1016/j.hrthm.2004.06.003. PMID:15851200.

Magri, D., De Cecco, C.N., Piccirillo, G., Mastromarino, V., Serdoz, A., Muscogiuri, G., et al. 2014. Myocardial repolarization dispersion and late gadolinium enhancement in patients with hypertrophic cardiomyopathy. Circulation Journal : official journal of the Japanese Circulation Society 78(5): 1216-1223. PMID:24632791.

Maron, B.J. 2002. Hypertrophic cardiomyopathy: a systematic review. JAMA 287(10): 13081320. PMID:11886323.

Maron, B.J. 2010. Contemporary insights and strategies for risk stratification and prevention of sudden death in hypertrophic cardiomyopathy. Circulation 121(3): 445-456. doi: 10.1161/CIRCULATIONAHA.109.878579. PMID:20100987.

Maron, B.J., Doerer, J.J., Haas, T.S., Tierney, D.M., and Mueller, F.O. 2009. Sudden deaths in young competitive athletes: analysis of 1866 deaths in the United States, 1980-2006. Circulation 119(8): 1085-1092. doi: 10.1161/CIRCULATIONAHA.108.804617. PMID:19221222.

Maron, B.J., Gardin, J.M., Flack, J.M., Gidding, S.S., Kurosaki, T.T., and Bild, D.E. 1995. Prevalence of hypertrophic cardiomyopathy in a general population of young adults. Echocardiographic analysis of 4111 subjects in the CARDIA Study. Coronary Artery Risk Development in (Young) Adults. Circulation 92(4): 785-789. PMID:7641357. 
Maron, B.J., Leyhe, M.J., 3rd, Casey, S.A., Gohman, T.E., Lawler, C.M., Crow, R.S., et al. 2001. Assessment of QT dispersion as a prognostic marker for sudden death in a regional nonreferred hypertrophic cardiomyopathy cohort. The American Journal of Cardiology 87(1): 114-115, A119. PMID:11137847.

Maron, B.J., Maron, M.S., Lesser, J.R., Hauser, R.G., Haas, T.S., Harrigan, C.J., et al. 2008. Sudden cardiac arrest in hypertrophic cardiomyopathy in the absence of conventional criteria for high risk status. The American Journal of Cardiology 101(4): 544-547. doi: 10.1016/j.amjcard.2007.09.101. PMID:18312775.

McKeown, P.P., and Muir, A.R. 2013. Risk assessment in hypertrophic cardiomyopathy: contemporary guidelines hampered by insufficient evidence. Heart 99(8): 511-513. doi: 10.1136/heartjnl-2012-303363. PMID:23376948.

Michael, G., Xiao, L., Qi, X.Y., Dobrev, D., and Nattel, S. 2009. Remodelling of cardiac repolarization: how homeostatic responses can lead to arrhythmogenesis. Cardiovascular Research 81(3): 491-499. doi: 10.1093/cvr/cvn266. PMID:18826964.

Monserrat, L., Elliott, P.M., Gimeno, J.R., Sharma, S., Penas-Lado, M., and McKenna, W.J. 2003. Non-sustained ventricular tachycardia in hypertrophic cardiomyopathy: an independent marker of sudden death risk in young patients. Journal of the American College of Cardiology 42(5): 873-879. PMID: 12957435.

Nattel, S., Maguy, A., Le Bouter, S., and Yeh, Y.H. 2007. Arrhythmogenic ion-channel remodeling in the heart: heart failure, myocardial infarction, and atrial fibrillation. Physiological Reviews 87(2): 425-456. doi: 10.1152/physrev.00014.2006. PMID:17429037.

Olivotto, I., Cecchi, F., Poggesi, C., and Yacoub, M.H. 2012. Patterns of disease progression in hypertrophic cardiomyopathy: an individualized approach to clinical staging. Circulation. Heart Failure 5(4): 535-546. doi: 10.1161/CIRCHEARTFAILURE.112.967026. PMID:22811549.

Olivotto, I., Maron, M.S., Autore, C., Lesser, J.R., Rega, L., Casolo, G., et al. 2008. Assessment and significance of left ventricular mass by cardiovascular magnetic resonance in hypertrophic cardiomyopathy. Journal of the American College of Cardiology 52(7): 559-566. doi: 10.1016/j.jacc.2008.04.047. PMID:18687251.

Ostman-Smith, I., Wisten, A., Nylander, E., Bratt, E.L., Granelli, A., Oulhaj, A., et al. 2010. Electrocardiographic amplitudes: a new risk factor for sudden death in hypertrophic cardiomyopathy. European Heart Journal 31(4): 439-449. doi: 10.1093/eurheartj/ehp443. PMID:19897498.

Panikkath, R., Reinier, K., Uy-Evanado, A., Teodorescu, C., Hattenhauer, J., Mariani, R., et al. 2011. Prolonged Tpeak-to-Tend interval on the resting ECG is associated with increased risk of sudden cardiac death. Circulation. Arrhythmia and Electrophysiology 4(4): 441-447. doi: 10.1161/CIRCEP.110.960658. PMID:21593198.

Piccirillo, G., Magri, D., Matera, S., Magnanti, M., Torrini, A., Pasquazzi, E., et al. 2007. QT variability strongly predicts sudden cardiac death in asymptomatic subjects with mild or 
moderate left ventricular systolic dysfunction: a prospective study. European Heart Journal 28(11): 1344-1350. doi: 10.1093/eurheartj/ehl367. PMID:17101636.

Schwartz, P.J., Priori, S.G., Spazzolini, C., Moss, A.J., Vincent, G.M., Napolitano, C., et al. 2001. Genotype-phenotype correlation in the long-QT syndrome: gene-specific triggers for life-threatening arrhythmias. Circulation 103(1): 89-95. PMID:11136691.

Shimizu, M., Ino, H., Okeie, K., Yamaguchi, M., Nagata, M., Hayashi, K., et al. 2002. T-peak to T-end interval may be a better predictor of high-risk patients with hypertrophic cardiomyopathy associated with a cardiac troponin I mutation than QT dispersion. Clinical Cardiology 25(7): 335-339. PMID:12109867.

Spirito, P., Autore, C., Rapezzi, C., Bernabo, P., Badagliacca, R., Maron, M.S., et al. 2009. Syncope and risk of sudden death in hypertrophic cardiomyopathy. Circulation 119(13): 1703-1710. doi: 10.1161/CIRCULATIONAHA.108.798314. PMID:19307481.

Spirito, P., Bellone, P., Harris, K.M., Bernabo, P., Bruzzi, P., and Maron, B.J. 2000. Magnitude of left ventricular hypertrophy and risk of sudden death in hypertrophic cardiomyopathy. The New England Journal of Medicine 342(24): 1778-1785. doi: 10.1056/NEJM200006153422403. PMID:10853000.

Thomsen, M.B., Verduyn, S.C., Stengl, M., Beekman, J.D., de Pater, G., van Opstal, J., et al. 2004. Increased short-term variability of repolarization predicts d-sotalol-induced torsades de pointes in dogs. Circulation 110(16): 2453-2459. doi: 10.1161/01.CIR.0000145162.64183.C8. PMID:15477402.

Tomaselli, G.F., and Marban, E. 1999. Electrophysiological remodeling in hypertrophy and heart failure. Cardiovascular Research 42(2): 270-283. PMID:10533566.

Valdivia, C.R., Chu, W.W., Pu, J., Foell, J.D., Haworth, R.A., Wolff, M.R., et al. 2005. Increased late sodium current in myocytes from a canine heart failure model and from failing human heart. Journal of Molecular and Cellular Cardiology 38(3): 475-483. doi: 10.1016/j.yjmcc.2004.12.012. PMID:15733907.

van Opstal, J.M., Schoenmakers, M., Verduyn, S.C., de Groot, S.H., Leunissen, J.D., van Der Hulst, et al. 2001. Chronic amiodarone evokes no torsade de pointes arrhythmias despite QT lengthening in an animal model of acquired long-QT syndrome. Circulation 104(22): 27222727. PMID:11723026.

Varkevisser, R., Wijers, S.C., van der Heyden, M.A., Beekman, J.D., Meine, M., and Vos, M.A. 2012. Beat-to-beat variability of repolarization as a new biomarker for proarrhythmia in vivo. Heart Rhythm: the official journal of the Heart Rhythm Society 9(10): 1718-1726. doi: 10.1016/j.hrthm.2012.05.016. PMID:22609158.

Varro, A., and Baczko, I. 2011. Cardiac ventricular repolarization reserve: a principle for understanding drug-related proarrhythmic risk. British Journal of Pharmacology 164(1): 1436. doi: 10.1111/j.1476-5381.2011.01367.x. PMID:21545574.

Vriesendorp, P.A., Schinkel, A.F., de Groot, N.M., van Domburg, R.T., Ten Cate, F.J., and Michels, M. 2014. Impact of adverse left ventricular remodeling on sudden cardiac death in 
patients with hypertrophic cardiomyopathy. Clinical Cardiology 37(8): 493-498. doi: 10.1002/clc.22293. PMID:25044226.

Wigle, E.D., Rakowski, H., Kimball, B.P., and Williams, W.G. 1995. Hypertrophic cardiomyopathy. Clinical spectrum and treatment. Circulation 92(7): 1680-1692. PMID:7671349.

Yamaguchi, M., Shimizu, M., Ino, H., Terai, H., Uchiyama, K., Oe, K., et al. 2003. T wave peak-to-end interval and QT dispersion in acquired long QT syndrome: a new index for arrhythmogenicity. Clinical Science 105(6): 671-676. doi: 10.1042/CS20030010. PMID:12857349.

Yi, G., Elliott, P., McKenna, W.J., Prasad, K., Sharma, S., Guo, X.H., et al. 1998. QT dispersion and risk factors for sudden cardiac death in patients with hypertrophic cardiomyopathy. The American Journal of Cardiology 82(12): 1514-1519. PMID:9874057.

Zeng, J., and Rudy, Y. 1995. Early afterdepolarizations in cardiac myocytes: mechanism and rate dependence. Biophysical Journal 68(3): 949-964. doi: 10.1016/S0006-3495(95)80271-7. PMID:7538806. 
Table 1. The main demographical, clinical and echocardiographic parameters of the patients with HCM and controls.

\begin{tabular}{|c|c|c|}
\hline & Control & HCM \\
\hline $\mathbf{n}$ & 37 & 37 \\
\hline Sex (male/female) & $21 / 16$ & $21 / 16$ \\
\hline Age (year) & $43 \pm 12$ & $48 \pm 15$ \\
\hline BMI (kg/m²) & $25 \pm 4$ & $28 \pm 6^{*}$ \\
\hline $\mathrm{BMI} \geq 30 / \geq 25 /<25 \mathrm{~kg} / \mathrm{m}^{2}$ (n) & $5 / 13 / 19$ & $11 / 16 / 10$ \\
\hline NYHA class $0 / 1 / 2 / 3(n)$ & $19 / 18 / 0 / 0$ & $0 / 5 / 26 / 6 * * * *$ \\
\hline EF $(\%)$ & $68 \pm 6$ & $69 \pm 9$ \\
\hline LVEDD (mm) & $48 \pm 4$ & $46 \pm 7$ \\
\hline LVESD (mm) & $30 \pm 4$ & $27 \pm 7^{*}$ \\
\hline IVS (mm) & $9 \pm 1$ & $20 \pm 6^{* * *}$ \\
\hline PW (mm) & $9 \pm 1$ & $11 \pm 2 * * *$ \\
\hline
\end{tabular}

Note: Values are represented as mean \pm SD. Values are considered statistically significantly different at $\mathrm{p}<0.05(*), \mathrm{p}<0.001(* * *)$ or $\mathrm{p}<0.0001(* * * *)$ compared with the control group. HCM, hypertrophic cardiomyopathy; BMI, body mass index; NYHA, New York Heart Association; EF, left ventricular ejection fraction; LVEDD, left ventricular end-diastolic diameter; LVESD, left ventricular end-systolic diameter; IVS, interventricular septum thickness; PW, posterior wall thickness. 
Table 2. Electrocardiographic parameters in HCM patients and controls.

\begin{tabular}{|c|c|c|c|}
\hline & Control & HCM & $\begin{array}{c}\text { Relative } \\
\text { difference }(\%)\end{array}$ \\
\hline RR (ms) & $867 \pm 119$ & $947 \pm 140 *$ & 9.2 \\
\hline PQ (ms) & $152 \pm 17$ & $166 \pm 30^{*}$ & 8.5 \\
\hline QRS (ms) & $96 \pm 7$ & $112 \pm 17 * * *$ & 15.6 \\
\hline QT (ms) & $401 \pm 24$ & $473 \pm 66^{* * *}$ & 18.0 \\
\hline QTc (ms) Bazett & $434 \pm 23$ & $488 \pm 61 * * *$ & 13.0 \\
\hline QTc (ms) Fridericia & $422 \pm 20$ & $483 \pm 60 * * *$ & 14.7 \\
\hline QTe (ms) Framingham & $423 \pm 19$ & $481 \pm 60 * * *$ & 14.5 \\
\hline QTe (ms) Hodges & $420 \pm 18$ & $482 \pm 60 * * *$ & 15.0 \\
\hline QTd (ms) & $34 \pm 9$ & $47 \pm 17 * *$ & 37.1 \\
\hline Tpeak-Tend (ms) & $91 \pm 10$ & $107 \pm 27^{*}$ & 18.9 \\
\hline QT-STV (ms) & $3.2 \pm 1$ & $4.5 \pm 2 * *$ & 40.6 \\
\hline
\end{tabular}

Note: Values are represented as mean \pm SD and relative difference between the two groups in $\%$. Values are considered statistically significantly different at $\mathrm{p}<0.05(*), \mathrm{p}<0.001(* *)$, $\mathrm{p}<0.0001(* * *)$ compared with the control group. $\mathrm{n}=37$ in each group. HCM, hypertrophic cardiomyopathy; QTc, heart rate correction of QT intervals (calculated by the Bazett's, Fridericia, Framingham and Hodges formulas); QTd, QT dispersion; Tpeak-Tend, duration of the T wave from the peak to the end; QT-STV, beat-to-beat short-term temporal variability of the QT interval. 
Table 3. Correlation of repolarization parameters in HCM patients.

\begin{tabular}{ccccc}
\hline & QRS & Tpeak-Tend & QTd & QT-STV \\
\hline QTe & 0.284 & $0.527 * * *$ & $-\mathbf{0 . 0 1 3}$ & $0.616^{* * *}$ \\
Tpeak-Tend & 0.299 & - & 0.018 & $0.378^{*}$ \\
QTd & 0.253 & - & - & $-\mathbf{0 . 2 2 8}$ \\
\hline
\end{tabular}

Note: Values are represented as Pearson correlation coefficient. Values are considered statistically significantly different at $\mathrm{p}<0.05(*), \mathrm{P}<0.001(* * *) ; \mathrm{n}=37$. HCM, hypertrophic cardiomyopathy; QTc, heart rate correction of QT intervals (calculated by the Bazett's formula); QTd, QT dispersion; Tpeak-Tend, duration of the T wave from the peak to the end; QT-STV, beat-to-beat short-term temporal variability of the QT interval. 
Table 4. Correlation between repolarization parameters and morphologic parameters of hypertrophy in HCM patients.

\begin{tabular}{|c|c|c|c|c|}
\hline & QTe & Tpeak-Tend & QTd & QT-STV \\
\hline IVS (mm) & 0.099 & $0.344^{*}$ & -0.144 & 0.285 \\
\hline LVmax (mm) & 0.216 & $0.450 * *$ & -0.238 & $0.381 *$ \\
\hline $\operatorname{LVmax} \operatorname{BSA}\left(\mathrm{mm} / \mathrm{m}^{2}\right)$ & $0.360 *$ & $0.451 * *$ & -0.129 & $0.461 * *$ \\
\hline LVM (g) & 0.037 & 0.241 & -0.128 & 0.273 \\
\hline LVM BSA $\left(\mathrm{g} / \mathrm{m}^{2}\right)$ & 0.195 & 0.348 & -0.116 & $0.455^{*}$ \\
\hline
\end{tabular}

Note: Values are represented as Pearson correlation coefficient. Values are considered statistically significantly different at $\mathrm{p}<0.05(*), \mathrm{p}<0.01(* *) ; \mathrm{n}=37$. HCM, hypertrophic cardiomyopathy; QTc, heart rate correction of QT intervals (calculated by the Bazett's formula); Tpeak-Tend, duration of the T wave from the peak to the end; QTd, QT dispersion; QT-STV, beat-to-beat short-term temporal variability of the QT interval; IVS, interventricular septum; LVmax, maximal left ventricular wall thickness; LVmax BSA, maximal left ventricular wall thickness normalized for body surface area; LVM, left ventricular mass; LVM BSA, left ventricular mass normalized for body surface area. 


\section{Figure captions}

Figure 1. Box and whisker plots illustrating significant differences between patients with HCM and controls with regard to A) the frequency corrected QT interval (QTc); B) the duration of terminal part of T waves (Tpeak-Tend); C) QT dispersion (QTd); D) beat-to-beat short-term variability of QT interval (QT-STV). The central box represents the values from the lower to upper quartile ( 25 to 75 percentile). The middle line represents the median. The vertical line extends from the minimum to the maximum value, excluding outside (open squares) and far out values (filled dots) which are displayed as separate points.

Figure 2. Scatterplot illustrating the correlation between maximal left ventricular wall thickness normalized for body surface area (LVmax BSA) and A) the frequency corrected QT interval (QTc); B) the duration of terminal part of T waves (Tpeak-Tend); C) QT dispersion (QTd); D) beat-to-beat short-term variability of QT interval (QT-STV).

Figure 3. Scatterplot illustrating the correlation between left ventricular mass normalized for body surface area (LVM BSA) and A) the frequency corrected QT interval (QTc); B) the duration of terminal part of T waves (Tpeak-Tend); C) QT dispersion (QTd); D) beat-to-beat short-term variability of QT interval (QT-STV). 

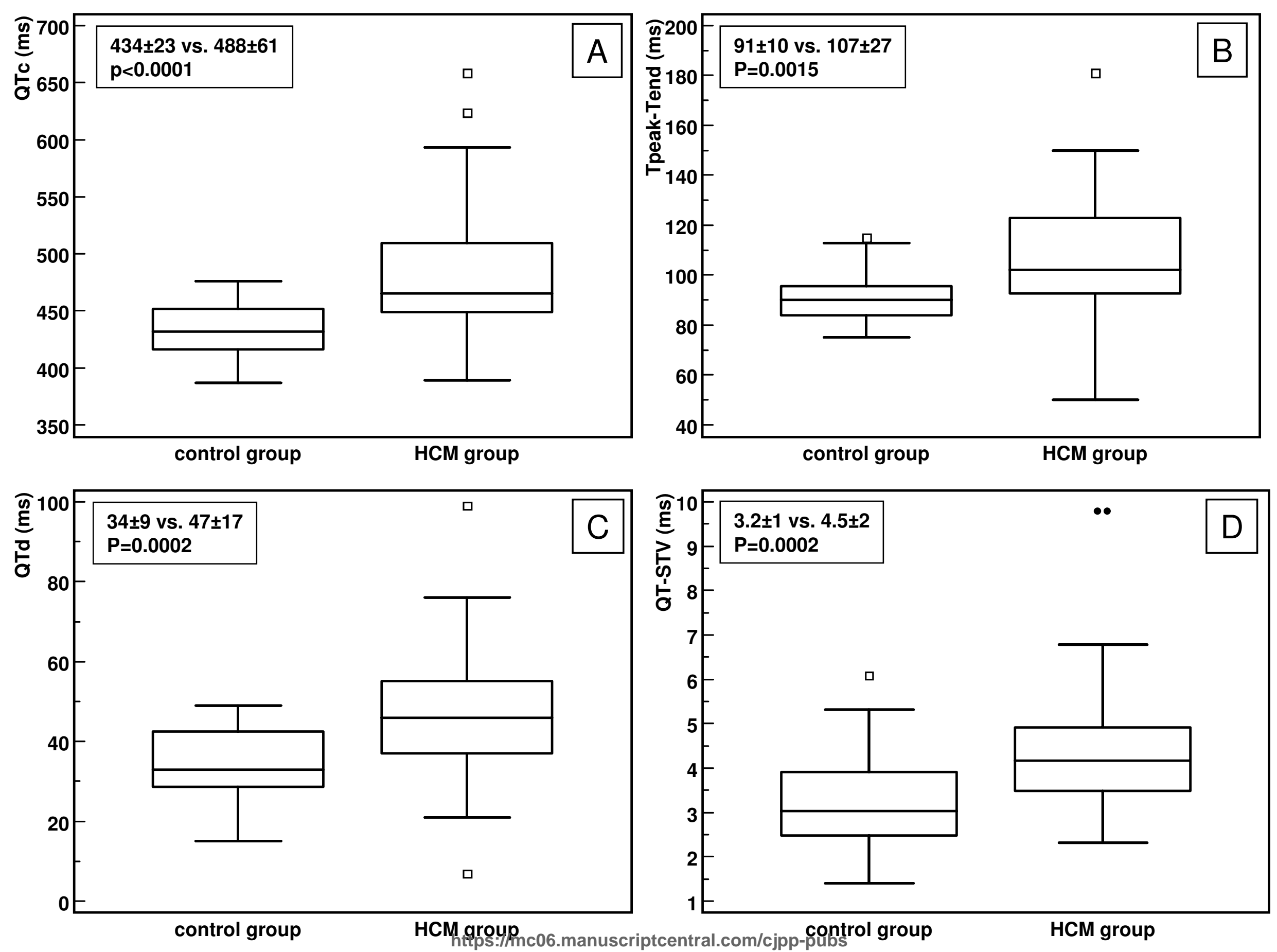

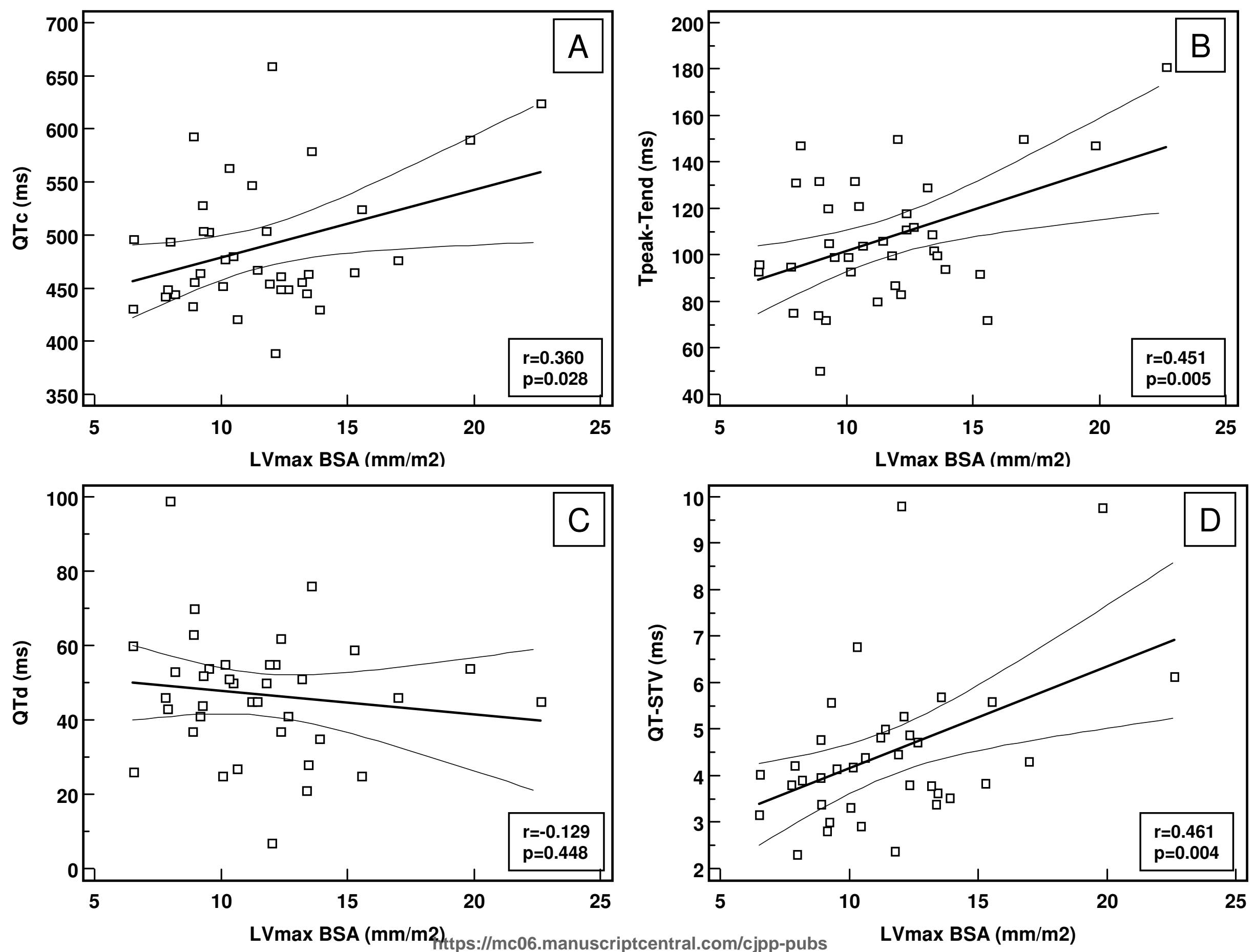

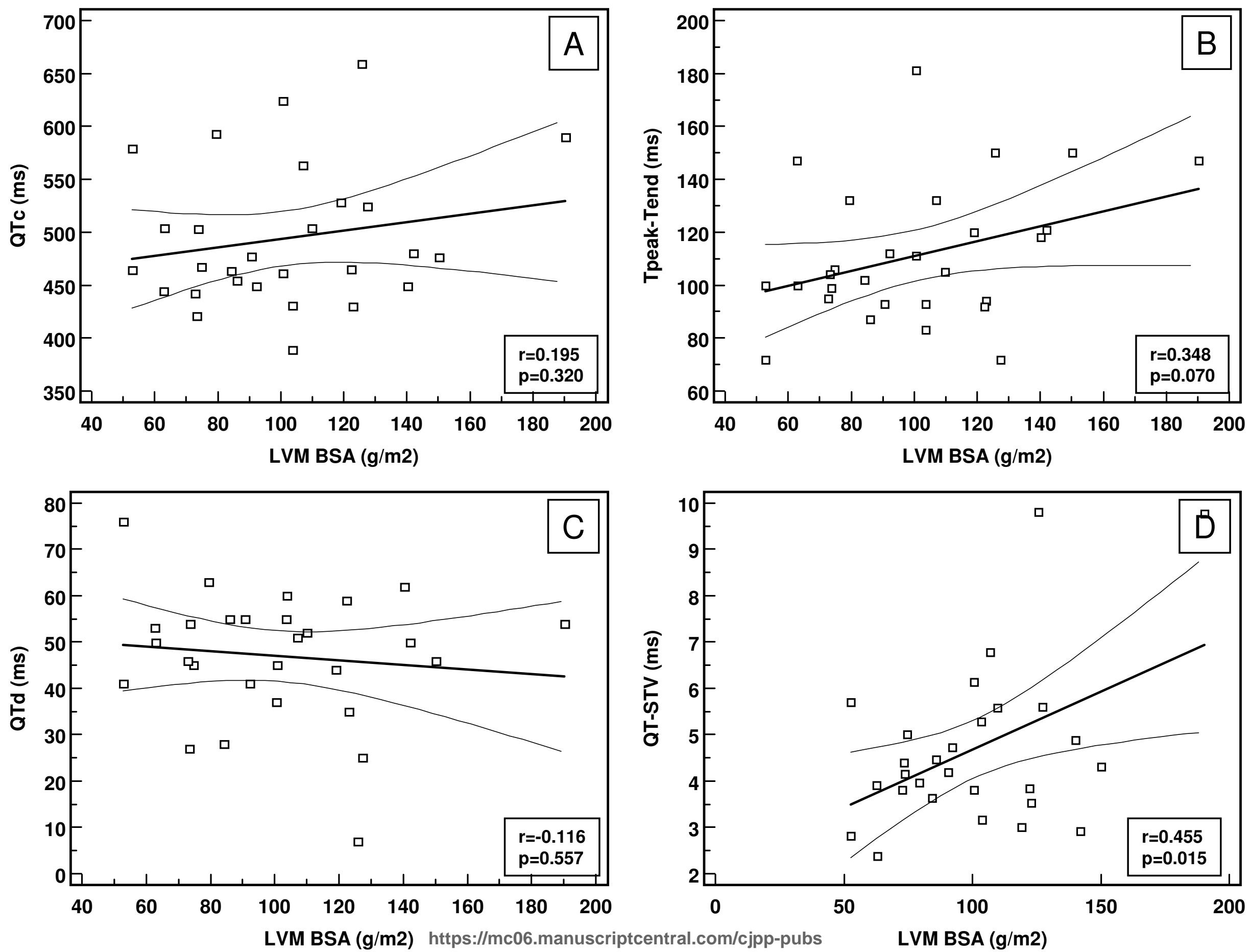